\title{
Comparison of the Generalized Riemann Solver and the Gas-Kinetic Scheme for Inviscid Compressible Flow Simulations
}

\author{
Jiequan $\mathrm{Li}^{\mathrm{a}}$, Qibing $\mathrm{Li}^{\mathrm{b}}, \mathrm{Kun} \mathrm{X} \mathrm{u}^{\mathrm{c}}$ \\ a School of Mathematical Science, Capital Normal University, Beijing, China \\ ${ }^{\mathrm{b}}$ Department of Engineering Mechanics, Tsinghua University, Beijing, China \\ ${ }^{\mathrm{c}}$ Department of Mathematics, Hong Kong University of Science and Technology, \\ Kowloon, Hong Kong
}

\begin{abstract}
The generalized Riemann problem (GRP) scheme for the Euler equations and gaskinetic scheme (GKS) for the Boltzmann equation are two high resolution shock capturing schemes for fluid simulations. The difference is that one is based on the characteristics of the inviscid Euler equations and their wave interactions, and the other is based on the particle transport and collisions. The similarity between them is that both methods can use identical MUSCL-type initial reconstructions around a cell interface, and the spatial slopes on both sides of a cell interface involve in the gas evolution process and the construction of a time-dependent flux function. Although both methods have been applied successfully to the inviscid compressible flow computations, their performances have never been compared. Since both methods use the same initial reconstruction, any difference is solely coming from different underlying mechanism in their flux evaluation. Therefore, such a comparison is important to help us to understand the correspondence between physical modeling and numerical performances. Since GRP is so faithfully solving the inviscid Euler equations, the comparison can be also used to show the validity of solving the Euler equations itself. The numerical comparison shows that the GRP exhibits a better computational efficiency, and has comparable accuracy with GKS for the Euler solutions in 1D case, but the GKS is more robust than GRP. For the 2D high Mach number flow simulations, the GKS is absent from the shock instability and converges to the steady state solutions faster than the GRP. The GRP has carbuncle phenomena, likes a cloud hanging over exact Riemann solver. The GRP and GKS use different physical processes to describe the flow motion starting from a discontinuity. One is based on the assumption of equilibrium state with infinite number of particle collisions, and the other starts from the non-equilibrium free transport process to evolve into an equilibrium one through particle collisions. The different mechanism in the flux evaluation deviates their performance. Through this study, we may conclude scientifically that it is NOT valid to use the Euler equations to construct numerical fluxes in a discretized space. To adapt Navier-Stokes (NS)
\end{abstract}

Preprint submitted to Elsevier

31 October 2010 
equations is NOT valid either because the NS has no any account on the strength of the discontinuity. A direct modeling of the physical process in the discretized space is necessary in the construction of numerical scheme. This process is similar to the modeling in deriving the governing equations, but the control volume here cannot be shrunk to zero.

Key words: Generalized Riemann problem scheme, gas kinetic scheme, Inviscid Euler equations, non-equilibrium flows

\section{Introduction}

The modern computational fluid dynamics (CFD) method for compressible flow is based on the Riemann problem from piecewise constant states [13]. The necessity to use discontinuous initial condition is due to the limited cell resolution to represent physical flow structure. Due to the preparation of discontinuous initial data through the so-called nonlinear limiter, the numerical dissipation is implicitly added in the shock capturing schemes. In the past decades, the shock capturing CFD methods based on the exact or approximate Riemann problems are extremely successful in the engineering applications and the scientific study of compressible flows. In order to further increase the accuracy, generalized Riemann solvers under piecewise linear discontinuous initial data were developed. The generalized Riemann problem (GRP) was proposed for compressible flows based on the Lagrangian formulation first $[2,4]$, and a direct Eulerian version was developed in [6,5] using the concept of Riemann invariants. Theoretically, in the GRP, a close coupling between the spatial and temporal evolution is recovered through the analysis of detailed wave interactions. The GRP is more precise and accurate than any other approximate Riemann solvers. It is the most accurate description of the Euler solutions under its corresponding initial condition. The schemes based on the GRP have been applied successfully to many engineering problems $[1,3,11,12,16]$.

On the other hand, based on the gas-kinetic theory, the Euler and Navier-Stokes equations can be derived from the Boltzmann equation using the Chapman-Enskog expansion [9]. In a gas-kinetic representation, all flow variables are moments of a single particle distribution function. In the past years, a gas-kinetic scheme (GKS) based on the BGK model [7] has been developed for the compressible flow simulation under linear polynomials for the mass, momentum, and energy distributions separated by discontinuities at the origin $[27,21]$. The GKS has been successfully applied in many engineering applications, especially for the hypersonic viscous and heat conducting flows $[28,18,19]$.

In this paper, we are going to compare the performance of GRP and GKS in many 1D and 2D flow computations, especially to the test cases on which many other schemes may have

Email addresses: jiequan@mail.cnu.edu.cn (Jiequan Li), lqb@tsinghua.edu.cn (Qibing Li), makxu@ust.hk (Kun Xu). 
difficulties, e.g. the large density ratio problem [23], highly expansion wave, and the blunt body problem. Since GRP and GKS use the identical initial reconstruction and follow explicitly their time evolution within a time step, any difference between these two methods must come from the dynamic mechanism in their flux construction. The GRP and GKS are following different physical process in the description of flow motion starting from a discontinuity. Their comparison definitely gives us useful information about the correspondence between the physical modeling and numerical performance. This is also the main reason why we do not use other Riemann solvers. For many other Riemann solvers, the problem is that either the real governing equations are unknown, or they are constructed in an ad hoc ways. The GRP truthfully follows the Euler solution and it has the most accurate Euler flux under the corresponding initial condition.

Through the comparison and analysis, we could like to deliver the following message. For algorithm development, the direct physical modeling of the gas evolution process in a discretized space is more fundamental and important than adapting any presumed governing equations from the start point. The Euler equations can be only used to describe the equilibrium flow, which is incapable to describe the non-equilibrium effects inside a numerical shock layer, especially for the strong shock case. In other words, the physical process in the GRP is inappropriate in the non-equilibrium region. As a result, the carbuncle phenomenon is intrinsically rooted in this scheme. Since GRP is so accurate in solving the Euler equations, we conclude that the direct use of the Euler equations in a discretized space is physically invalid. The GKS provides a more reliable gas evolution model than GRP to describe flow starting from a discontinuity. We hope that through this study and any other following up research, the CFD will evolve from an art-based to a science-based research subject.

\section{Generalized Riemann Problem and Gas-kinetic Schemes}

In this paper we will focus on the comparison of numerical results for the compressible Euler equations,

$$
\begin{aligned}
& \mathbf{u}_{t}+\mathbf{F}(\mathbf{u})_{x}+\mathbf{G}(\mathbf{u})_{y}=0, \\
& \mathbf{u}=(\rho, \rho U, \rho V, \rho E)^{\top}, \\
& \mathbf{F}(\mathbf{u})=\left(\rho U, \rho U^{2}+p, \rho U V, U(\rho E+p)\right)^{\top}, \\
& \mathbf{G}(\mathbf{u})=\left(\rho V, \rho U V, \rho V^{2}+p, V(\rho E+p)\right)^{\top},
\end{aligned}
$$

where $\rho,(\rho U, \rho V)$ and $\rho E$ are conserved variables of the density, the momentum and the total energy with $E=\frac{U^{2}+V^{2}}{2}+e, e$ the internal energy, $p$ is the pressure and $p=(\gamma-1) \rho e$ 
for polytropic gases. Then following the finite volume formulation, we write (1) as

$$
\mathbf{u}_{j}^{n+1}=\mathbf{u}_{j}^{n}-\frac{1}{\left|\Omega_{j}\right|} \sum_{i=1}^{m} \int_{t^{n}}^{t^{n+1}} F_{i j} d t
$$

where $\Omega_{i}$ is the control volume with sides $\Gamma_{i j}, j=1, \cdots, m(m=4$ in this paper), $\mathbf{n}=$ $\left(n_{j 1}, n_{j 2}\right)$ is the outer normal of $\Gamma_{i j}$, and the numerical flux $F_{i j}$ is

$$
F_{i j}=\int_{\Gamma_{i j}}(\mathbf{F}, \mathbf{G})(\mathbf{u}) \cdot\left(n_{i 1}, n_{i 2}\right) d \Gamma,
$$

where $\mathbf{u}_{j}^{n}$ is the usual average of $\mathbf{u}\left(x, t_{n}\right)$ over $\Omega_{j}$. In one-dimensional case, $\Omega_{j}=\left(x_{j-\frac{1}{2}}, x_{j+\frac{1}{2}}\right)$ and the center $x_{j}=\left(x_{j-\frac{1}{2}}+x_{j+\frac{1}{2}}\right) / 2$.

The GRP and GKS both provide a respective time-dependent flux function from a piecewise linear discontinuous initial data. In this section, we are going to first present the reconstruction used in the present paper.

As expressed in (2), at the beginning of each time step $t=t_{n}$ only cell averaged mass, momentum and energy densities are given. As far as a high-resolution scheme is concerned, interpolation techniques are used to construct the subcell structure. Simple polynomials usually generate spurious oscillations if large gradients exist in the data. The most successful interpolation techniques known so far are those based on the concepts of limiters [8,25], and these interpolation rules can be applied to the conservative, characteristic or primitive flow variables. In this paper, the reconstruction is solely applied to the conservative variables. The limiter used is the van Leer limiter. With the cell averaged component of conservative variables $w_{j}$, and their differences between neighboring cells $s_{+}=\left(w_{j+1}-w_{j}\right) / \Delta x$ and $s_{-}=\left(w_{j}-w_{j-1}\right) / \Delta x$, the slope of $w$ in cell $j$ is constructed as

$$
L\left(s_{+}, s_{-}\right)=S\left(s_{+}, s_{-}\right) \frac{\left|s_{+}\right|\left|s_{-}\right|}{\left|s_{+}\right|+\left|s_{-}\right|},
$$

where $S\left(s_{+}, s_{-}\right)=\operatorname{sign}\left(s_{+}\right)+\operatorname{sign}\left(s_{-}\right)$. After reconstruction, the flow variable $w$ is distributed linearly in cell $j$,

$$
\bar{w}_{j}(x)=w_{j}+L\left(s_{+}, s_{-}\right)\left(x-x_{j}\right), \quad x_{j-\frac{1}{2}}<x<x_{j+\frac{1}{2}} .
$$

The interpolated flow distribution around a cell interface is shown in Fig.1. Both the GRP and GKS are based on the above initial data to evaluate a time-dependent local solution. 


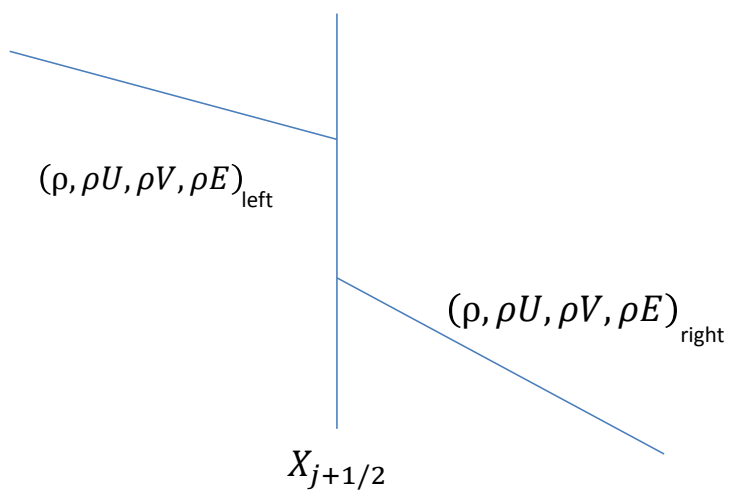

Fig. 1. The reconstructed initial conservative variables around a cell interface.

\subsection{The Generalized Riemann Problem for the inviscid Euler Equations}

The generalized Riemann problem (GRP) scheme starts directly from (1). Denote by $P_{i j}$ the middle point of $\Gamma_{i j}$ and $F_{i j}=(\mathbf{F}, \mathbf{G}) \cdot\left(n_{i 1}, n_{i 2}\right)$. Then the GRP uses the middle point value to directly approximate the flux $F_{i j}^{n+\frac{1}{2}}$, within the accuracy of second order,

$$
F_{i j}^{n+\frac{1}{2}} \sim F_{i j}\left(\mathbf{u}\left(P_{i j},\left(n+\frac{1}{2}\right) \Delta t\right)\right) .
$$

One of main issues is how to calculate the mid-point value $\mathbf{u}\left(P_{i j},\left(n+\frac{1}{2}\right) \Delta t\right)$ using as much characteristics of (1) as possible. Here we use the Taylor series expansion,

$$
\mathbf{u}\left(P_{i j},\left(n+\frac{1}{2}\right) \Delta t\right)=\mathbf{u}\left(P_{i j}, t_{n}+0\right)+\frac{\Delta t}{2} \frac{\partial \mathbf{u}}{\partial t}\left(P_{i j}, t_{n}+0\right)+\mathcal{O}\left(\Delta t^{2}\right) .
$$

Fix the cell interface $\Gamma_{i j}$ and denote by

$$
\mathbf{u}_{*}=\mathbf{u}\left(P_{i j}, t_{n}+0\right), \quad\left(\frac{\partial \mathbf{u}}{\partial t}\right)_{*}=\frac{\partial \mathbf{u}}{\partial t}\left(P_{i j}, t_{n}+0\right) \text {. }
$$

The first instantaneous value $\mathbf{u}_{*}$ is obtained with the standard Riemann solver [4,24]. The main contribution of the GRP scheme is how to obtain the instantaneous value of time derivative $(\partial \mathbf{u} / \partial t)_{*}$ analytically, which boils down to solving the associated generalized Riemann problem along each cell interface $\Gamma_{i j}$. For this purpose, we shift $\left(P_{i j}, t_{n}\right)$ to the coordinate origin $(0,0,0)$ and make $\Gamma_{i j}$ coincide with the $y$-axis. Then we define the quasi-one-dimensional (planar) generalized Riemann problem for

$$
\mathbf{u}_{t}+\mathbf{F}(\mathbf{u})_{x}=0
$$


subject to the initial data

$$
\mathbf{u}(x, y, 0)=\left\{\begin{array}{l}
\mathbf{u}_{\ell}+x \mathbf{u}_{x \ell}^{\prime}+y \mathbf{u}_{y \ell}^{\prime}, x<0, \\
\mathbf{u}_{r}+x \mathbf{u}_{x r}^{\prime}+y \mathbf{u}_{y r}^{\prime}, x>0
\end{array}\right.
$$

where $\mathbf{u}_{\ell}, \mathbf{u}_{x \ell}^{\prime}, \mathbf{u}_{y \ell}^{\prime}, \mathbf{u}_{r}, \mathbf{u}_{x r}^{\prime}, \mathbf{u}_{y r}^{\prime}$ are constant vectors. In particular, as $\mathbf{u}_{y \ell}^{\prime}$ and $\mathbf{u}_{y r}^{\prime}$ vanishes, Eq.(8) becomes planar one-dimensional flows. The conventional GRP solver uses the planar one-dimensional Euler equations (7) for simplicity and efficiency. Then we assume the wave configuration from the $y$-axis as shown in Fig. 2, in which a curved rarefaction wave moves to the left, a shock to the right and the $t$-axis is located in the intermediate region. Other cases can be treated similarly. We remind that as the initial data is uniform, i.e., piecewise constant, the rarefaction wave in Fig. 2 becomes isentropic, referred as a centered rarefaction wave $(\mathrm{CRW})$. Then the instantaneous value of time derivative $(\partial \mathbf{u} / \partial t)_{*}$ can be solved in the following proposition.

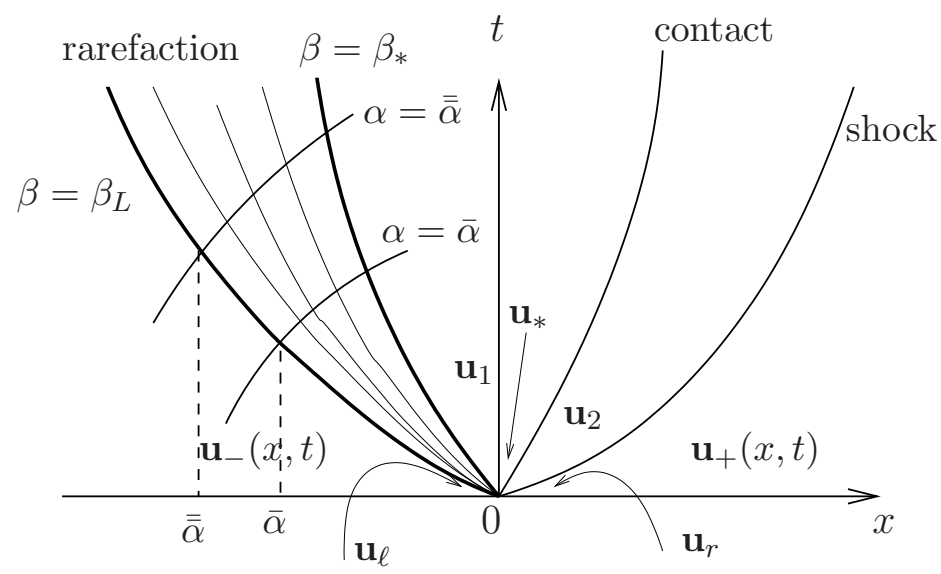

Fig. 2. A typical wave pattern for the GRP. The initial data $\mathbf{u}_{0}(x)=\mathbf{u}_{\ell}+x \mathbf{u}_{\ell}^{\prime}$ for $x<0$ and $\mathbf{u}_{0}(x)=\mathbf{u}_{r}+x \mathbf{u}_{r}^{\prime}$ for $x>0$.

Proposition 1 Let $(\partial \mathbf{u} / \partial t)_{*}$ be the instantaneous time derivative of $\mathbf{u}$ evaluated at the origin. Then $(\partial U / \partial t)_{*}$ and $(\partial p / \partial t)_{*}$ are determined by solving a pair of linear equations

$$
\begin{aligned}
& a_{\ell}\left(\frac{\partial U}{\partial t}\right)_{*}+b_{\ell}\left(\frac{\partial p}{\partial t}\right)_{*}=d_{\ell} \\
& a_{r}\left(\frac{\partial U}{\partial t}\right)_{*}+b_{r}\left(\frac{\partial p}{\partial t}\right)_{*}=d_{r}
\end{aligned}
$$

where the coefficients $a_{\ell}, b_{\ell}$ and $d_{\ell}$ depends on $\mathbf{u}_{*}, \mathbf{u}_{\ell}$ and $\mathbf{u}_{\ell}^{\prime}$; and $a_{r}, b_{r}$ and $d_{r}$ depends on $\mathbf{u}_{*}, \mathbf{u}_{r}$ and $\mathbf{u}_{r}^{\prime}$. The instantaneous time derivative of the density $\rho$ is given by the state equation $p=p(\rho, S)$, $S$ is the entropy,

$$
d p=c^{2} d \rho+\frac{\partial p}{\partial S} d S .
$$


The velocity component $V$ satisfies

$$
V_{t}+U V_{x}=0
$$

It resembles the entropy variable $S$ (cf. (17) below) and can be treated similarly.

All these coefficients can be obtained explicitly as presented in [6]. It turns out that the coding process is almost the same as a $2 \times 2$ linear system that we will show below.

In the derivation of this proposition, the fundamental idea is to extract singularities (discontinuities) from the complex wave patterns:

$$
\text { Wave patterns }=\text { a singular part }+ \text { a regular part. }
$$

The GRP scheme expresses and manipulates the regular part using Riemann invariants and leave the singular part aside. This manipulation is indispensable. Below is a heuristic example of a linear toy system

$$
\begin{aligned}
& u_{t}+a v_{x}=0, \\
& v_{t}+a u_{x}=0,
\end{aligned}
$$

where $a>0$ is a constant. In consistent with the notations in $(8), \mathbf{u}=(u, v)^{\top}$ here. In order to develop the second order MUSCL-type (upwind) scheme for (13), the piecewise linear data is given

$$
\mathbf{u}(x, 0)= \begin{cases}\mathbf{u}_{\ell}+x \mathbf{u}_{\ell}^{\prime}, & x<0, \\ \mathbf{u}_{r}+x \mathbf{u}_{r}^{\prime}, & x>0,\end{cases}
$$

and we need to know the intermediate value $\mathbf{u}(0, t)$ along the $t$-axis. Note that the initial data contains a discontinuity at the origin, causing nontrivial singularities (discontinuities) that propagate along characteristics $x= \pm$ at, which implies that both the variables $u$ and $v$ are discontinuous across $x= \pm a t$. In order to cope with this difficulty, it is necessary to extract out the singularities in some sense. For this purpose, we make the following manipulation for (13)

$$
\begin{aligned}
& (u+v)_{t}+a(u+v)_{x}=0, \\
& (u-v)_{t}-a(u-v)_{x}=0 .
\end{aligned}
$$


Then the assembled quantity $u+v$ is continuous in the region $\{(x, t) ; x<a t, t>0\}$ although both $u$ and $v$ are discontinuous across $x=-$ at. The same is true for $u-v$ in the region $\{(x, t) ; x>-a t, t>0\}$. Thus we use $u+v$ and $u-v$ as new dependent variables to easily obtain

$$
\begin{gathered}
u_{*}+v_{*}=u_{\ell}+v_{\ell}, \quad u_{*}-v_{*}=u_{r}-v_{r} \\
\left(\frac{\partial u}{\partial t}\right)_{*}+\left(\frac{\partial v}{\partial t}\right)_{*}=-a\left(u_{\ell}^{\prime}+v_{\ell}^{\prime}\right), \quad\left(\frac{\partial u}{\partial t}\right)_{*}-\left(\frac{\partial v}{\partial t}\right)_{*}=a\left(u_{r}^{\prime}-v_{\ell}^{\prime}\right) .
\end{gathered}
$$

Then, in turn, we obtain $\mathbf{u}_{*}$ and $(\partial \mathbf{u} / \partial t)_{*}$ by solving the pairs of linear equations (16).

This procedure has several implications:

(1) The assembled quantities $u+v$ and $u-v$, corresponding to the Riemann invariants for (1), are used to achieve the claim (12). More precisely, take a look at the characteristic $x=-a t$, across which, as mentioned above, both $u$ and $v$ are discontinuous. However, $u+v$ is regular there and the singularity along $x=-a t$ has been removed solely onto $u-v$. Then we can manipulate $u+v$ as a scalar variable for a single linear advection equation.

(2) In smooth regions, the Lax-Wendroff approach can be used to define the instantaneous time derivatives of (proper) assembled variables, which are replaced by the spatial derivatives.

(3) The resulting pair of linear equations are then solved to obtain the instantaneous time derivatives of primitive variables finally.

It seems that this is the only reasonable way to extend a scalar case to the system (13) in order to construct the numerical flux with the least numerical dissipation under the second order MUSCL-type initial condition.

The GRP methodology for (1) uses almost the same idea: look for Riemann invariants, extract singularities, and derive a pair of linear equations (9), particularly in the resolution of rarefaction waves. Since the entropy variable is necessarily taken as one of Riemann invariants and exactly computed, the rarefaction wave can be well resolved so that the resulting scheme is able to capture both (even very strong) rarefaction and shocks waves.

Let us illuminate this in some detail. We fix the case of the left-moving rarefaction wave associated with the characteristic field $U-c, c$ is the sound speed, as shown in figure 2 . The primitive variables $(\rho, U, p)$ (or the conservative variables $(\rho, \rho U, \rho E)$ ) are singular through the rarefaction wave, particularly around the origin. Use the Riemann invariants $\psi(\rho, U, p)=U+\frac{2 c}{\gamma-1}$ and $S$ (for polytropic gases, and similarly for general gases) to write 
(1) in terms of $\psi$ and $S$ as

$$
\begin{aligned}
& \psi_{t}+(U+c) \psi_{x}=T S_{x} \\
& S_{t}+U S_{x}=0
\end{aligned}
$$

where $T$ is the temperature. Since $\psi$ keeps invariant across the centered isentropic rarefaction wave (CRW) in the associated Riemann problem, it behaves exactly like $u+v$ for (13) and be smooth through the entire curved rarefaction wave in the generalized Riemann problem. Therefore, we can use them to designate the regular part of the rarefaction wave. The singular part is removed into another variable $\phi=U-\frac{2 c}{\gamma-1}$ that satisfies

$$
\phi_{t}+(U-c) \phi_{x}=T S_{x}
$$

Note that $\phi$ is also a Riemann invariant but associated with another characteristic field $U+c$. We resolve (17) to obtain the first equation of (9).

The second equation of (9) can obtained through tracing the right-going shock trajectory, following van Leer's idea [25].

The source terms in (17) and (18) reflect the variation of entropy as the initial state is not uniform. This variation is well captured in the GRP scheme, and then the variable $\psi$ is resolved subsequently in an analytical way, using the Lax-Wendroff approach. Due to the regularity of $\psi$ and $S$ and their analytical resolution, it is expected that the rarefaction wave can be captured very accurately using the GRP scheme, and it is indeed. The detail can be found in $[6,5]$.

\subsection{The gas-kinetic scheme}

In this section, a directional splitting method to solve the 2D BGK equation will be presented. The BGK model in the $x$-direction can be written as [9]

$$
f_{t}+u f_{x}=\frac{g-f}{\tau}
$$

where $f$ is the gas distribution function, $g$ is the equilibrium state approached by $f$, and $\tau$ is the particle collision time. Both $f$ and $g$ are functions of space $x$, time $t$, particle velocities $(u, v)$, and internal variable $\xi$. The particle collision time $\tau$ is related to the viscosity and heat conduction coefficients. The equilibrium state is a Maxwellian distribution,

$$
g=\rho\left(\frac{\lambda}{\pi}\right)^{\frac{K+2}{2}} e^{-\lambda\left((u-U)^{2}+(v-V)^{2}+\xi^{2}\right)},
$$


where $\rho$ is the density, $U$ and $V$ are the macroscopic velocities in the $x$ and $y$ directions, as in (1), and $\lambda=m / 2 k T$, where $m$ is the molecular mass, $k$ is the Boltzmann constant, and $T$ is the temperature. For a 2D flow with explicit account of $U$ and $V$ velocities, the random particle motion in the $z$ direction is included into the internal variable $\xi$, and the total number of degrees of freedom $K$ in $\xi$ is equal to $(5-3 \gamma) /(\gamma-1)+1$. In the equilibrium state, the internal variable $\xi^{2}$ is equal to $\xi^{2}=\xi_{1}^{2}+\xi_{2}^{2}+\ldots+\xi_{K}^{2}$. The relation between mass $\rho$, momentum $(n=\rho U, m=\rho V)$, and energy $\rho E$ densities with the distribution function $f$ is

$$
\left(\begin{array}{c}
\rho \\
n \\
m \\
\rho E
\end{array}\right)=\int \psi_{\alpha} f d \Xi, \quad \alpha=1,2,3,4
$$

where $\psi_{\alpha}$ is the component of the vector of moments

$$
\boldsymbol{\psi}=\left(\psi_{1}, \psi_{2}, \psi_{3}, \psi_{4}\right)^{\top}=\left(1, u, v, \frac{1}{2}\left(u^{2}+v^{2}+\xi^{2}\right)\right)^{\top}
$$

and $d \Xi=d u d v d \xi$ is the volume element in the phase space with $d \xi=d \xi_{1} d \xi_{2} \ldots d \xi_{K}$. Since mass, momentum and energy are conserved during particle collisions, $f$ and $g$ satisfy the conservation constraint,

$$
\int(g-f) \psi_{\alpha} d \Xi=0, \quad \alpha=1,2,3,4
$$

at any point in space and time. For a local equilibrium state with $f=g$, the Euler equations can be obtained by taking the moments of $\psi_{\alpha}$ to Eq.(19). This yields

$$
\int \psi_{\alpha}\left(g_{t}+u g_{x}\right) d \Xi=0, \quad \alpha=1,2,3,4,
$$

and the corresponding Euler equations in the $x$-direction are (1).

The general solution $f$ of the BGK model (19) at a cell interface $x_{j+1 / 2}$ and time $t$ is

$$
f\left(x_{j+1 / 2}, t, u, v, \xi\right)=\frac{1}{\tau} \int_{0}^{t} g\left(x^{\prime}, t^{\prime}, u, v, \xi\right) e^{-\left(t-t^{\prime}\right) / \tau} d t^{\prime}+e^{-t / \tau} f_{0}\left(x_{j+1 / 2}-u t\right),
$$

where $x^{\prime}=x_{j+1 / 2}-u\left(t-t^{\prime}\right)$ is the particle trajectory and $f_{0}$ is the initial gas distribution function $f$ at the beginning of each time step $(t=0)$. Two unknowns $g$ and $f_{0}$ must be specified in Eq.(22) in order to obtain the solution $f$. In order to simplify the notation, $x_{j+1 / 2}=0$ will be used in the following text. Based on the MUSCL-type reconstruction for 
the macroscopic variables at a cell interface, the initial gas distribution function $f_{0}$ has the corresponding form,

$$
f_{0}=\left\{\begin{array}{ll}
g^{l}\left[1+a^{l} x-\tau\left(a^{l} u+A^{l}\right)\right], & x \leq 0 \\
g^{r}\left[1+a^{r} x-\tau\left(a^{r} u+A^{r}\right)\right], & x \geq 0
\end{array},\right.
$$

where the terms being proportional to $\tau$ are the nonequilibrium states obtained from the Chapman-Enskog expansion of the BGK model. The parameters of $\left(a^{l}, A^{l}, a^{r}, A^{r}\right)$ are related to the Taylor expansion of a Maxwellian. The nonequilibrium parts have no direct contribution to the conservative variables, i.e.,

$$
\begin{aligned}
\int\left(a^{l} u+A^{l}\right) \boldsymbol{\psi} g^{l} d \Xi & =0, \\
\int\left(a^{r} u+A^{r}\right) \boldsymbol{\psi} g^{r} d \Xi & =0 .
\end{aligned}
$$

After having $f_{0}$, the equilibrium state $g$ around $(x=0, t=0)$ is assumed to have two slopes,

$$
g=g_{0}\left[1+(1-\mathrm{H}[x]) \bar{a}^{l} x+\mathrm{H}[x] \bar{a}^{r} x+\bar{A} t\right],
$$

where $\mathrm{H}[x]$ is the Heaviside function defined as

$$
H[x]= \begin{cases}0, & x<0 \\ 1 . & x \geq 0\end{cases}
$$

Here $g_{0}$ is a local Maxwellian distribution function located at $x=0$. Even though, $g$ is continuous at $x=0$, but it has different slopes at $x<0$ and $x>0$. In both $f_{0}$ and $g$, $a^{l}, A^{l}, a^{r}, A^{r}, \bar{a}^{l}, \bar{a}^{r}$, and $\bar{A}$ are related to the derivatives of Maxwellians in space and time. Detailed formulation can be found in [27].

In the reconstruction stage, we have obtained the distributions $\bar{\rho}_{j}(x), \bar{m}_{j}(x), \bar{n}_{j}(x)$, and $\rho_{j} \bar{E}_{j}(x)$ inside each cell $x_{j-1 / 2} \leq x \leq x_{j+1 / 2}$. At the cell interface $x_{j+1 / 2}$, the left and right macroscopic states are

$$
\mathbf{u}_{j}\left(x_{j+1 / 2}\right)=\left(\begin{array}{c}
\bar{\rho}_{j}\left(x_{j+1 / 2}\right) \\
\bar{m}_{j}\left(x_{j+1 / 2}\right) \\
\bar{n}_{j}\left(x_{j+1 / 2}\right) \\
\bar{\rho} \bar{E}_{j}\left(x_{j+1 / 2}\right)
\end{array}\right) \quad, \quad \mathbf{u}_{j+1}\left(x_{j+1 / 2}\right)=\left(\begin{array}{c}
\bar{\rho}_{j+1}\left(x_{j+1 / 2}\right) \\
\bar{m}_{j+1}\left(x_{j+1 / 2}\right) \\
\bar{n}_{j+1}\left(x_{j+1 / 2}\right) \\
\bar{\rho} \bar{E}_{j+1}\left(x_{j+1 / 2}\right)
\end{array}\right)
$$

By using the relation between the gas distribution function $f$ and the macroscopic variables (Eq.(20)), at $x_{j+1 / 2}$ we get

$$
\int g^{l} \boldsymbol{\psi} d \Xi=\mathbf{u}_{j}\left(x_{j+1 / 2}\right) ; \int g^{l} a^{l} \boldsymbol{\psi} d \Xi=\frac{\mathbf{u}_{j}\left(x_{j+1 / 2}\right)-\mathbf{u}_{j}\left(x_{j}\right)}{\Delta x^{-}}
$$




$$
\int g^{r} \boldsymbol{\psi} d \Xi=\mathbf{u}_{j+1}\left(x_{j+1 / 2}\right) ; \int g^{r} a^{r} \boldsymbol{\psi} d \Xi=\frac{\mathbf{u}_{j+1}\left(x_{j+1}\right)-\mathbf{u}_{j+1}\left(x_{j+1 / 2}\right)}{\Delta x^{+}}
$$

where $\Delta x^{-}=x_{j+1 / 2}-x_{j}$ and $\Delta x^{+}=x_{j+1}-x_{j+1 / 2}$. Based on the above equations, all parameters in the initial distribution function $f_{0}$ can be fully determined.

After determining $f_{0}$, the corresponding values of $\rho_{0}, U_{0}, V_{0}$ and $\lambda_{0}$ in $g_{0}$ of Eq.(25), i.e.,

$$
g_{0}=\rho_{0}\left(\frac{\lambda_{0}}{\pi}\right)^{\frac{K+2}{2}} e^{-\lambda_{0}\left(\left(u-U_{0}\right)^{2}+\left(v-V_{0}\right)^{2}+\xi^{2}\right)},
$$

can be determined as follows. Taking the limit $t \rightarrow 0$ in Eq.(22) and substituting its solution into Eq.(21), the conservation constraint at $\left(x=x_{j+1 / 2}, t=0\right)$ gives

$$
\int g_{0} \boldsymbol{\psi} d \Xi=\mathbf{u}_{0}=\int_{u>0} \int g^{l} \boldsymbol{\psi} d \Xi+\int_{u<0} \int g^{r} \boldsymbol{\psi} d \Xi
$$

where $\mathbf{u}_{0}=\left(\rho_{0}, m_{0}, n_{0}, \rho_{0} E_{0}\right)^{T}$ are the macroscopic conservative flow variables located at the cell interface at time $t=0$.

Then, $\bar{a}^{l}$ and $\bar{a}^{r}$ of $g$ in Eq.(25) can be obtained through the relation of

$$
\frac{\mathbf{u}_{j+1}\left(x_{j+1}\right)-\mathbf{u}_{0}}{\rho_{0} \Delta x^{+}}=\bar{M}_{\alpha \beta}^{0}\left(\begin{array}{c}
\bar{a}_{1}^{r} \\
\bar{a}_{2}^{r} \\
\bar{a}_{3}^{r} \\
\bar{a}_{4}^{r}
\end{array}\right)=\bar{M}_{\alpha \beta}^{0} \bar{a}_{\beta}^{r},
$$

and

$$
\frac{\mathbf{u}_{0}-\mathbf{u}_{j}\left(x_{j}\right)}{\rho_{0} \Delta x^{-}}=\bar{M}_{\alpha \beta}^{0}\left(\begin{array}{c}
\bar{a}_{1}^{l} \\
\bar{a}_{2}^{l} \\
\bar{a}_{3}^{l} \\
\bar{a}_{4}^{l}
\end{array}\right)=\bar{M}_{\alpha \beta}^{0} \bar{a}_{\beta}^{l},
$$

where the matrix $\bar{M}_{\alpha \beta}^{0}=\int g_{0} \psi_{\alpha} \psi_{\beta} d \Xi / \rho_{0}$ is known.

Up to this point, we have determined all parameters in the initial gas distribution function $f_{0}$ and the equilibrium state $g$ at the beginning of each time step $t=0$. After substituting Eq.(23) and Eq.(25) into Eq.(22), the gas distribution function $f$ at a cell interface can be expressed as

$$
\begin{aligned}
f\left(x_{j+1 / 2}, t, u, v, \xi\right)= & \left(1-e^{-t / \tau}\right) g_{0} \\
& +\left(\tau\left(-1+e^{-t / \tau}\right)+t e^{-t / \tau}\right)\left(\bar{a}^{l} \mathrm{H}[u]+\bar{a}^{r}(1-\mathrm{H}[u])\right) u g_{0}
\end{aligned}
$$




$$
\begin{aligned}
& +\tau\left(t / \tau-1+e^{-t / \tau}\right) \bar{A} g_{0} \\
& +e^{-t / \tau}\left(\left(1-u(t+\tau) a^{l}\right) \mathrm{H}[u] g^{l}+\left(1-u(t+\tau) a^{r}\right)(1-\mathrm{H}[u]) g^{r}\right) \\
& +e^{-t / \tau}\left(-\tau A^{l} \mathrm{H}[u] g^{l}-\tau A^{r}(1-\mathrm{H}[u]) g^{r}\right) .
\end{aligned}
$$

The only unknown left in the above expression is $\bar{A}$. Since both $f$ (Eq.(31)) and $g$ (Eq.(25)) contain $\bar{A}$, the integration of the conservation constraint Eq.(21) at $x_{j+1 / 2}$ over the whole time step $\Delta t$ gives

$$
\int_{0}^{\Delta t} \int(g-f) \boldsymbol{\psi} d t d \Xi=0 .
$$

The evaluation of $\bar{A}$ is about to get time variation of macroscopic variables at a cell interface, which is similar to the GRP in the evaluations of $(\partial U / \partial t)_{*}$ and $(\partial p / \partial t)_{*}$.

Finally, the time-dependent numerical fluxes in the $x$-direction across the cell interface can be computed as

$$
\left(\begin{array}{c}
F_{\rho} \\
F_{m} \\
F_{n} \\
F_{\rho E}
\end{array}\right)_{j+1 / 2}=\int u\left(\begin{array}{c}
1 \\
u \\
v \\
\frac{1}{2}\left(u^{2}+v^{2}+\xi^{2}\right)
\end{array}\right) f\left(x_{j+1 / 2}, t, u, v, \xi\right) d \Xi
$$

where $f\left(x_{j+1 / 2}, t, u, v, \xi\right)$ is given in Eq.(31). By integrating the above equation to the whole time step, we can get the total mass, momentum and energy transport. For the Euler solutions, theoretically the particle collision time $\tau$ should approach to zero in order to keep the equilibrium state everywhere. However, the about formulation (31) describes a relaxation process, the Euler solution is only the limiting case in the GKS. Theoretically, the collision time $\tau$ is proportional to mean free path and particle transport characteristic speed. In a discretized sapce, due to cell resolution the particle mean free path can be assumed to be proportional to the cell size. Therefore, the collision time used in all test cases in this paper have the following form,

$$
\tau=\Delta x \sqrt{\lambda_{0}}\left(\alpha+\beta\left|p^{l}-p^{r}\right| /\left(p^{l}+p^{r}\right)\right)
$$

with $\alpha=0.05$ and $\beta=1.0$, where $\lambda_{0}$ is given in the equilibrium state $g_{0}$, and $p^{l}$ and $p^{r}$ are the pressure jump at the cell interface in the initial reconstructed data. The consideration for the above formulation is the following. As mesh size goes to zero, $\tau$ will go to zero as well, and the GKS converges to the Euler solutions.

In the smooth flow region, there are many numerical mean free paths in each cell size. However, in the dissipative shock region, the number of numerical mean free path is much 
reduced to capture the non-equilibrium effect, such as the real physical process inside a shock layer. This is equivalent to enlarging the physical shock thickness to numerical cell size scale, but with the similar particle transport and collision mechanism.

\section{Details of the test cases}

In this section we will present quite full comparison of numerical results by the GRP and GKS schemes. These examples are all very challenging, ranging from several one-dimensional blast wave problem to the high speed flow impinging on a cylinder. Unless otherwise stated, van Leer limiter is adopted and the CFL number is set to 0.5. Since GRP and GKS use the identical van Leer limiter for the conservative flow variables reconstruction, any difference in the simulation results is due to the flux function, i.e., the mechanism of gas evolution models around a cell interface. Since GRP is truthfully solving the Euler equations and the GKS is following the kinetic equation, the comparison distinguishes the effects of different physical processes on the simulation results.

\subsection{Problems in one-dimensional space}

\subsubsection{Woodward-Colella blast wave problem}

We first show the interacting blast wave problem proposed in [26]. The diatomic gas is initially at rest, and the density is everywhere unit. The pressure is $p=1000$ for $0 \leq x<10$ and $p=100$ for $90<x \leq 100$, while it is only $p=0.01$ in $10<x<90$. Reflecting boundary conditions are applied at both ends. Numerical results with 800 grid points are shown in Fig. 3 to exhibit the performance of both schemes. This test case clearly demonstrates the capability of both schemes in the capturing of strong shock waves. The GRP presents a little bit sharper solution than the GKS in the internal energy distribution.

\subsubsection{Low density problem}

This example was first proposed in [10] for demonstrating the ability of a numerical scheme to preserve the positivity of density and internal energy. In contrast to the blast wave problem, this test shows strong rarefaction waves. The initial data is given with $(\rho, U, p)=(1,2,0.4)$

for $0 \leq x<50$ and $(\rho, U, p)=(1,2,0.4)$ for $50 \leq x \leq 100$. The number of grid points used here is 100 . Both schemes work well, as shown in Fig. 4. Many approximate Riemann solvers may fail in this test case [10]. 


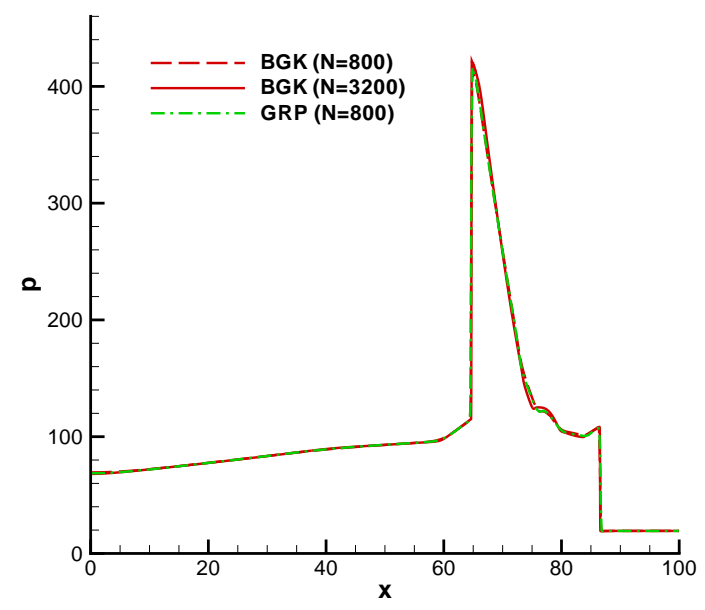

(a)

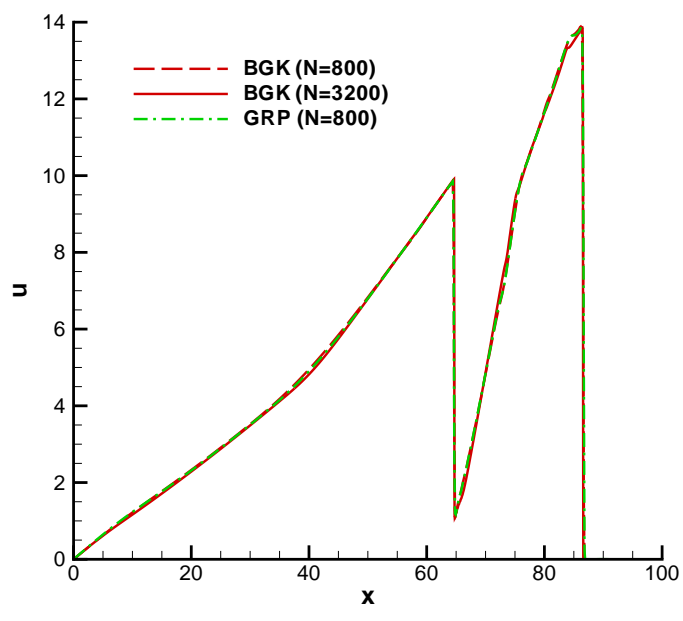

(b)

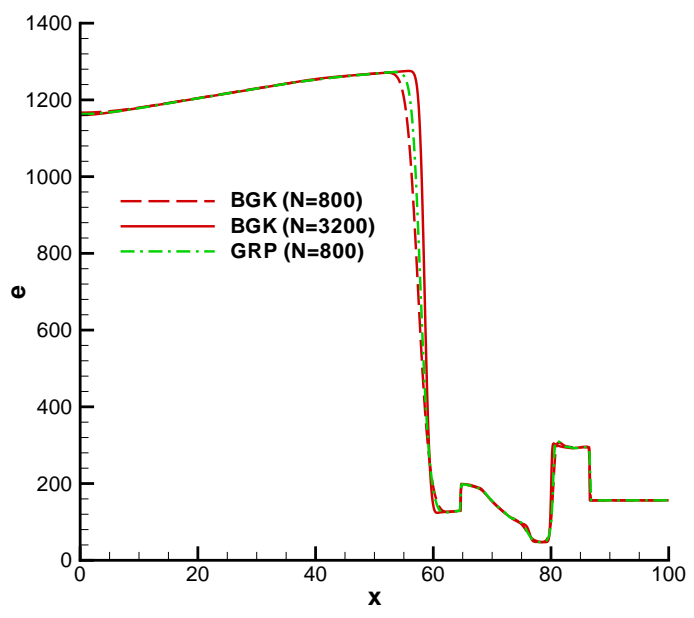

(c)

Fig. 3. Woodward-Colella blast wave problem. (a) pressure, (b) velocity, (c) internal energy.

\subsection{Large density ratio problem}

This example was proposed in [23] to test the ability to capture extremely strong rarefaction wave and its influence on the shock location. In the original paper, it shows that most MUSCL-type schemes have defects in resolving, even with very fine mesh, the correct wave structures. In this problem, initially the pressure and density ratio between two neighboring states are very high. The initial data is $(\rho, U, p)=(10000,0,10000)$ for $0 \leq x<30$ and $(\rho, U, p)=(1,0,1)$ for $30 \leq x \leq 100$. CFL number is set as 0.32 . The results with 100 and 400 points from both GRP and GKS are shown in Fig. 5. For GRP, from 100 to 400 grid points, the shock location converges to the correct position. With 400 grid points, the GRP scheme gives perfect results, which can be comparable to those from very high resolution 


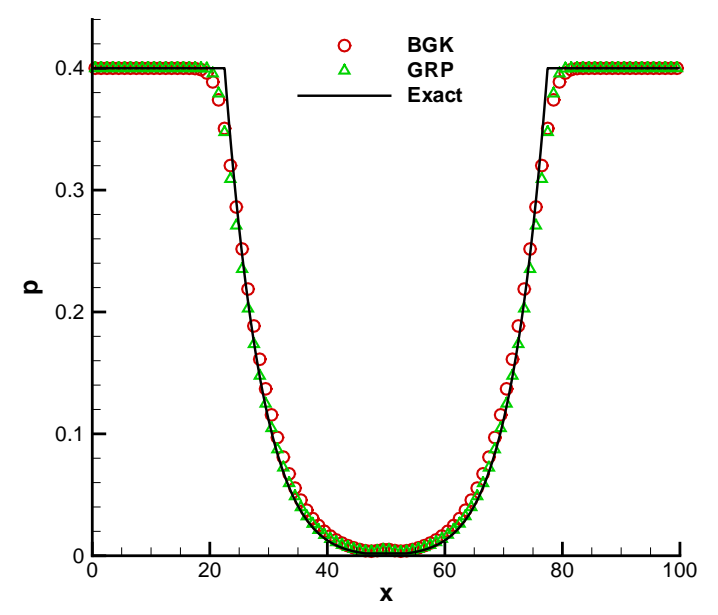

(a)

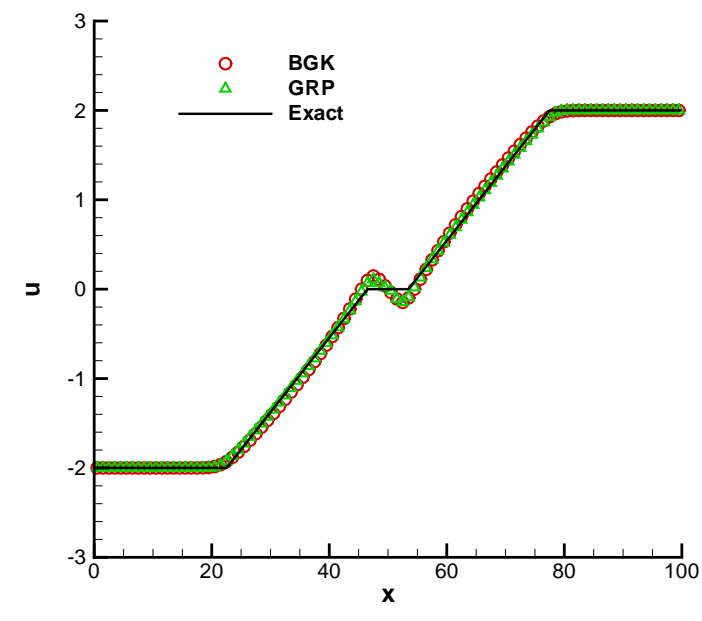

(b)

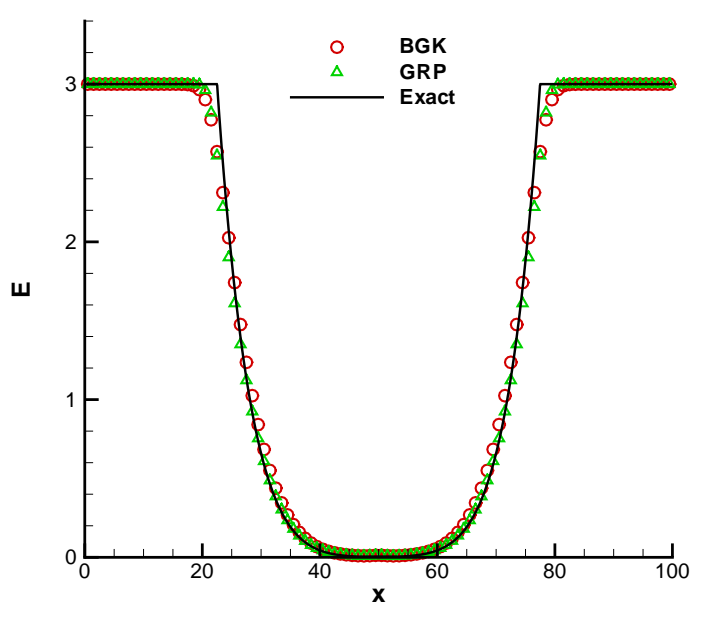

(c)

Fig. 4. Sjogreen's low density problem.(a) pressure, (b) velocity, (c) energy.

schemes, i.e., fifth order WENO scheme with 10000 grid points [23]. Surprisingly, the GKS presents the same shock front location with 100 and 400 grid points. One of the reason is that the GKS solves viscous governing equations, but the exact solution is the inviscid one. In order to clarify the situation, we reduce the particle collision time in Eq.(33) to $\alpha=\beta=10^{-4}$. With the reducing of dissipation, the GKS results with 100 and 400 grid points are shown in Fig. 6. Again, the GKS keeps almost the same shock location for 100 and 400 grid points. Even with 100 grid points, it can capture the solution very well. This clearly demonstrates that the GKS converges to the inviscid Euler solution as the particle collision time reduces. 


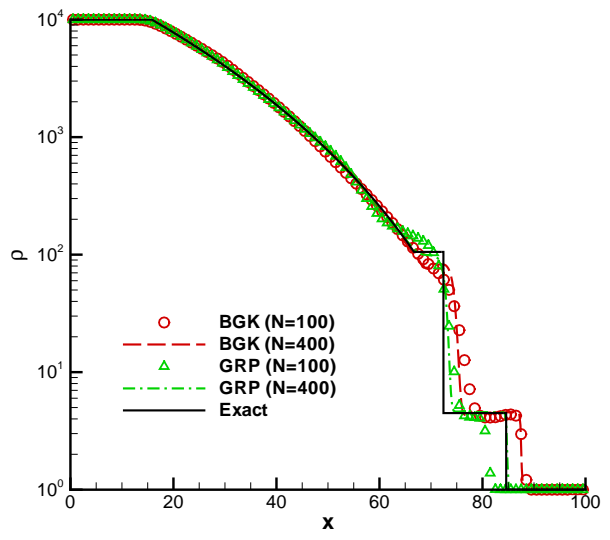

(a)

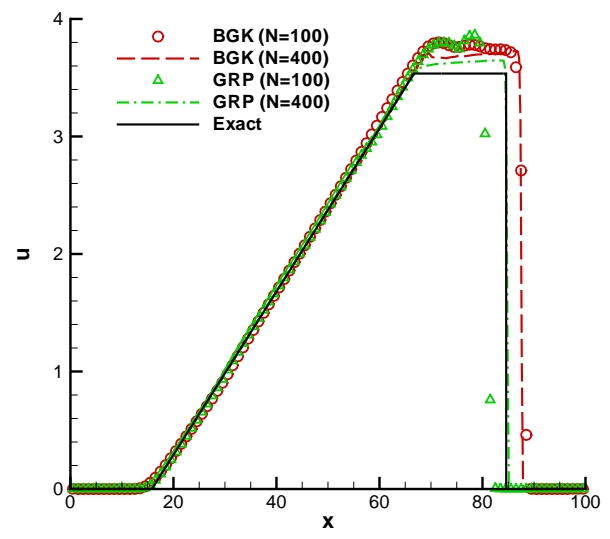

(c)

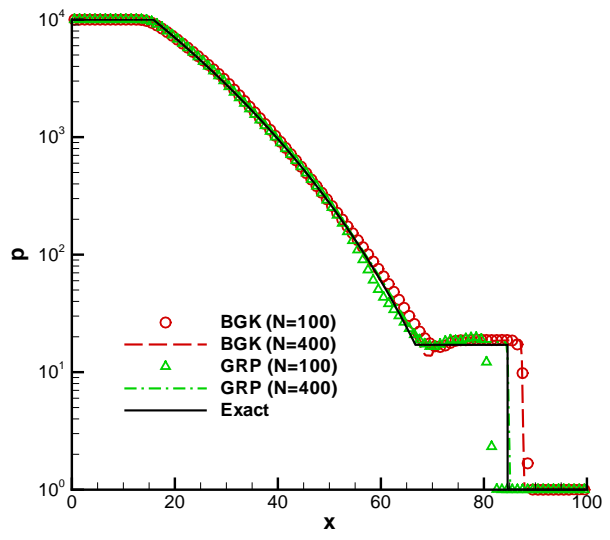

(b)

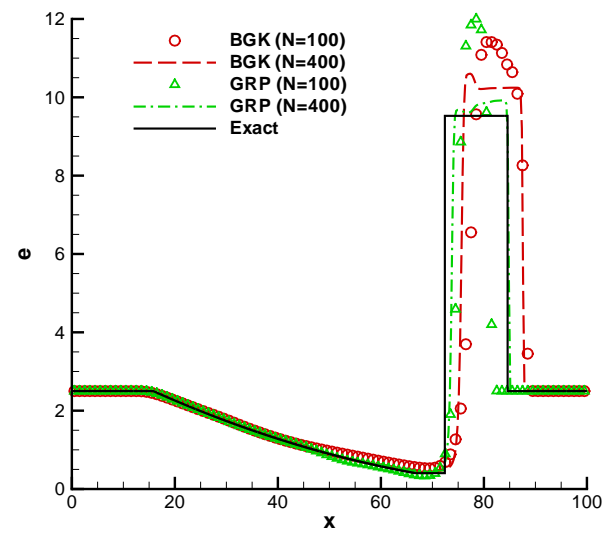

(d)

Fig. 5. Large density ration problem. (a) density, (b) pressure, (c) velocity, (d) internal energy.

\subsection{Slowly moving shock}

This is a test case for strong slowly moving shock with Minmod limiter for both schemes. The initial data is: $(\rho, U, p)=(4.0,0.3,4 / 3)$ for $0 \leq x<20$; and $(\rho, U, p)=\left(1.0,-1.3,10^{-6}\right)$ for $20<x \leq 100$. The polytropic index is taken to be $\gamma=5 / 3$. This is an almost infinite strong shock in the sense that the density ratio is close to its maximum.

The simulation results from both 100 and 400 grid points are shown in Fig. 7. The shock fronts are captured crisply by both schemes. In the coarse mesh with 100 grid points, the GKS has a better shock location. As the mesh is refined, both schemes converge to the exact solution. The GKS generates less magnitude of post-shock oscillation than GRP.

The above several examples are fully recognized as severe test problems and many shock 


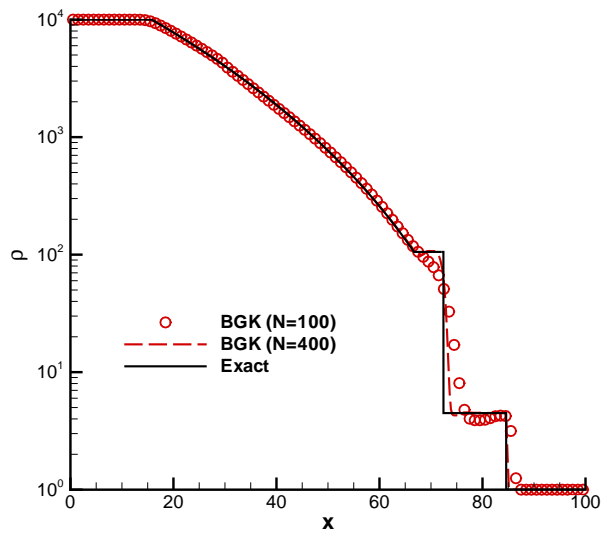

(a)

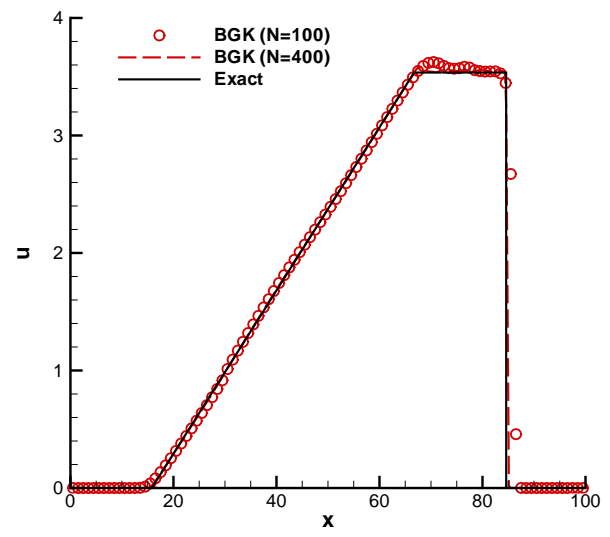

(c)

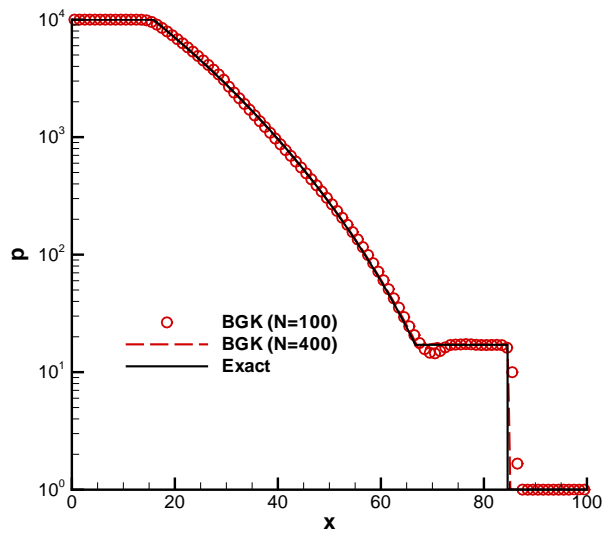

(b)

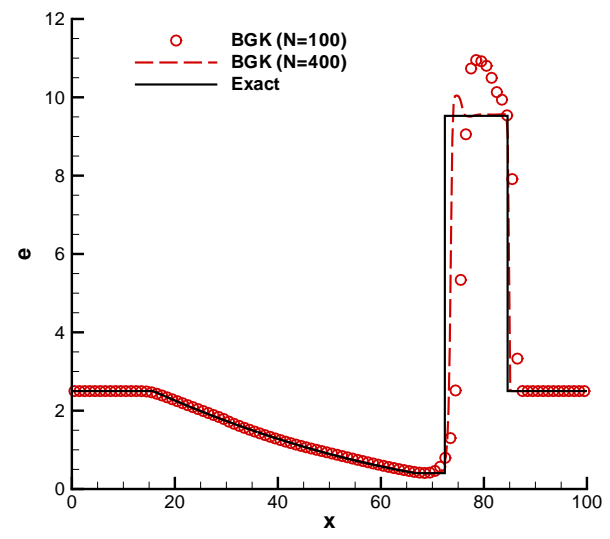

(d)

Fig. 6. Large density ration problem. (a) density, (b) pressure, (c) velocity, (d) internal energy. capturing schemes may fail to provide accurate or even correct results, such as the correct position of shock front in [23]. The GRP and GKS encounter no difficulties for these cases. One of the reason is that both schemes can compute the entropy accurately. As a result, the rarefaction wave is resolved perfectly, so is the shock location. Also, through the comparison we can realize that the accuracy of GKS in obtaining the inviscid Euler solutions even though it targets on the kinetic model.

\subsection{Problems in two-dimensions}

In comparison with the 1D test cases, two-dimensional simulations are much more interesting and challenging. The GRP is basically 1D gas evolution model. In order to make GKS be consistent with GRP, we adapt the direction splitting GKS here in all simulations. A multidimensional GKS scheme has been developed as well [28]. In the 2D case, some classical test problems such as double Mach reflection have been reported in [14,27] using these two 


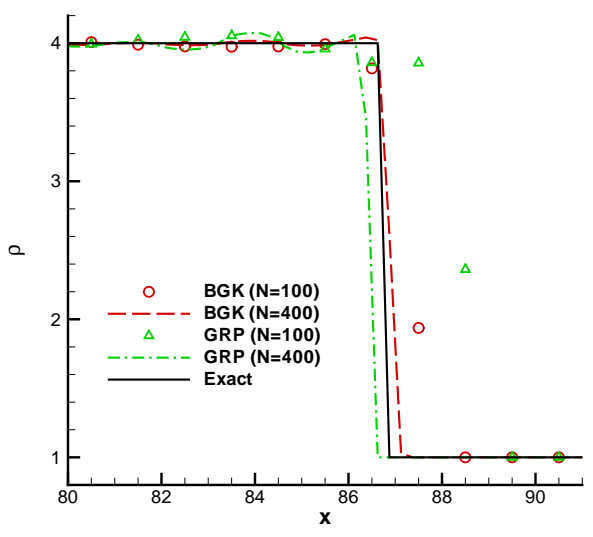

(a)

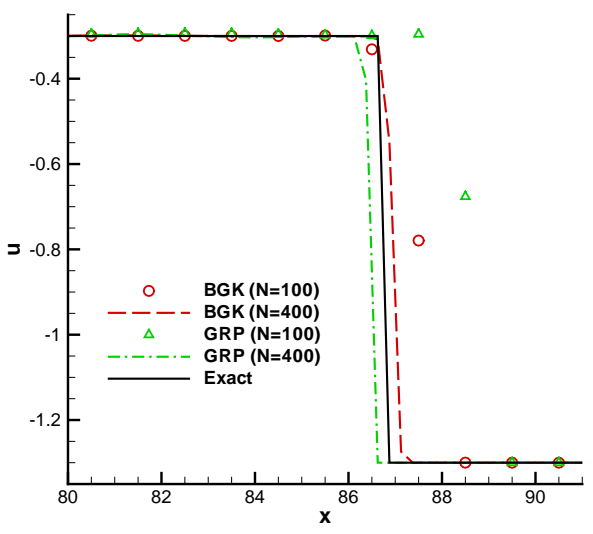

(c)

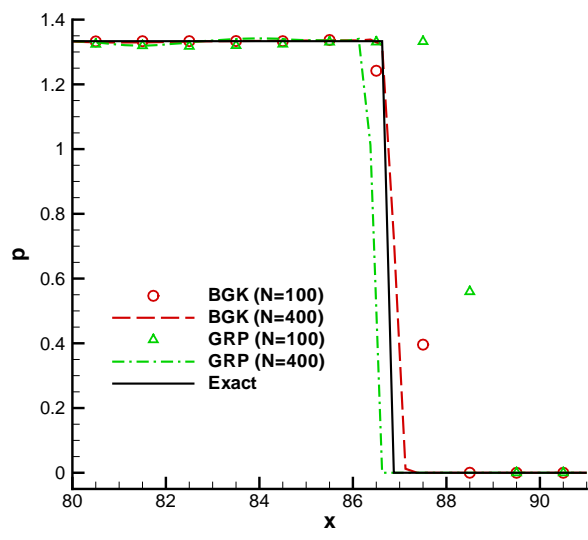

(b)

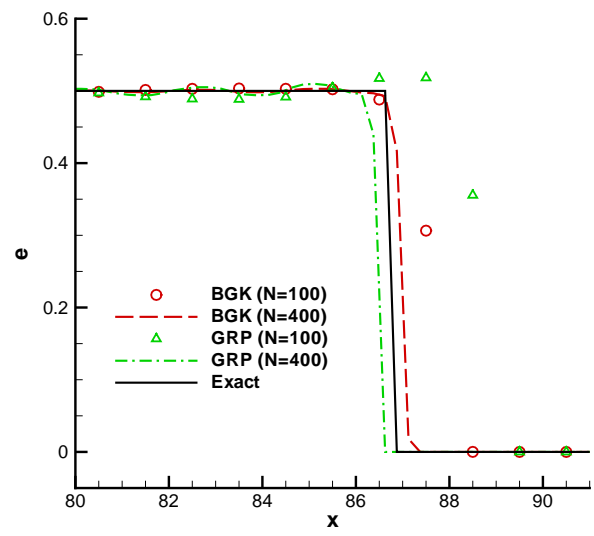

(d)

Fig. 7. Slowly moving shock problem. (a) density, (b) pressure, (c) velocity, (d) internal energy.

schemes. Here we choose two problems of interaction of (compressible) vortex sheets and the flow impinging on a cylinder. The CFL number is set to be 0.5 and 0.7 , respectively.

\subsubsection{Formation of spiral through the interaction of vortex sheets}

This is a special case of 2-D Riemann problems formulated in [29] to simulate the formation of spirals, see also $[17,14]$ for the solution structure. The initial data is a constant state in each quadrant: $(\rho, U, V, p)=(0.5,0.5,-0.5,5)$ for $x>0, y>0,(\rho, U, V, p)=$ $(1.0,0.5,0.5,5)$ for $x<0, y>0,(\rho, U, V, p)=(2.0,-0.5,0.5,5)$ for $x<0, y<0,(\rho, U, V, p)=$ $(1.5,-0.5,-0.5,5)$ for $x>0, y<0$. Initially four vortex sheets are supported, respectively, on the $x$ and $y$-axes with the same sign, but they have different amounts of measures. They interact immediately after $t>0$ to generate a spiral in the center and the state there closes to the vacuum. We can see the wonderful performance of both schemes from Fig. 8 by the comparison with those in $[22,20,15]$. 


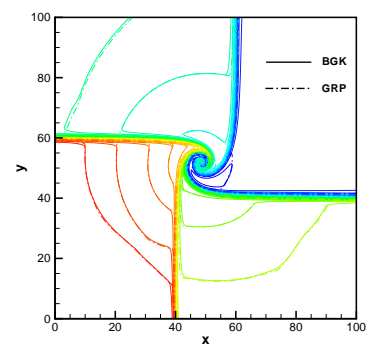

(a) The contour of density

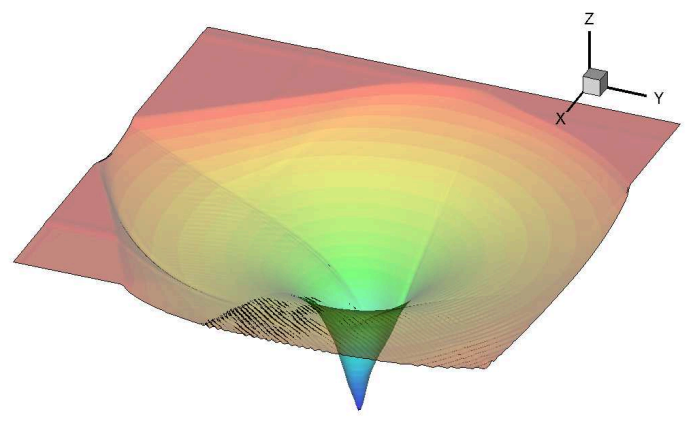

(b) Pressure distribution

Fig. 8. Formation of spiral in the 2D Riemann problem.

\subsubsection{Flow impinging on a cylinder}

For the blunt body simulations, we test flows with Mach number 2, 5 and 10. Also, three meshes have been used, which are shown in Fig.9. We call them mesh (a), (b), and (c). The difference between mesh (a) and (b) comes from the different size of the computational domains to account for the standoff distance of the bow shock. The length-width (circumferential-radial) ratio of Mesh (c) is a little smaller. At $M=2$ with mesh (a), GRP and GKS can capture the flow structure nicely in front of the cylinder, which are shown in Fig.10 for the Mach number and temperature distributions. The residual for both calculation is shown in Fig. 11. The GKS converges to machine zero quickly and the convergence of GRP is saturated at $10^{-4}$. At $M=5$ with mesh (b), the simulation results from GRP and GKS are shown in Fig.12 as well as the convergence rate in Fig. 13. At $M=5$, even with convergent results, GRP has difficulty in capturing a smooth solution in front of the cylinder. It seems that GRP converges to another solution. This may raise the question about the uniqueness of the Euler solution. For GKS, the same as $M=2$ case, it presents a nice result. As we increase the Mach number to 10, with the same mesh (b), the GKS and GRP results are shown in Fig. 14. Surprisingly, GRP presents a smooth solution in comparison with $M=5$ case, even though it still has wiggles around sonic lines. However, for the $M=10$ case, with mesh (c) the simulation results from GKS and GRP are shown in Fig. 15. Again, GRP presents oscillatory results and the calculations break down eventually. Even with the first order Godunov method, an oscillatory solutions will be obtained as well. In both $M=10$ calculation with different meshes, GKS converges to machine zero in the same way as $M=5$ case.

In the cylinder case, both GKS and GRP are using the identical mesh and reconstruction scheme. Any difference is coming from the flux evaluation. Since GRP is so truthfully solving the Euler equations under the corresponding initial condition, the simulation results clearly demonstrate the fundamental flaws in solving the inviscid Euler equations for the capturing of hypersonic flow in the blunt body calculation. Even with the fatal results from GRP, theoretically we do not know how to fix it, since the GRP is so accurately solving the Euler 
equations. In the cylinder case, the flow structure is relatively simple. The flaws in the GRP must come from its representation of the numerical shock layer in multi-dimensional case. As we know, with limited cell resolution, a physical shock structure is enlarged up to the mesh size scale. Inside the shock layer, a highly non-equilibrium state is present. This nonequilibrium state can be captured by following real physical process of particle transport and collisions. This is somehow consistent the mechanism underlying the GKS, where the free transport and collision are both included in the flux evaluation. However, from the starting point, GRP assumes an equilibrium state everywhere, even inside the numerical shock layer. GRP lacks the mechanism to construct a non-equilibrium shock layer on the mesh size scale, and it has inappropriate physical mechanism in its flux construction there. Therefore, it is fundamentally flawed to use the Euler equations as a foundation in the construction of shock capturing scheme. More analysis will be presented in the next section.

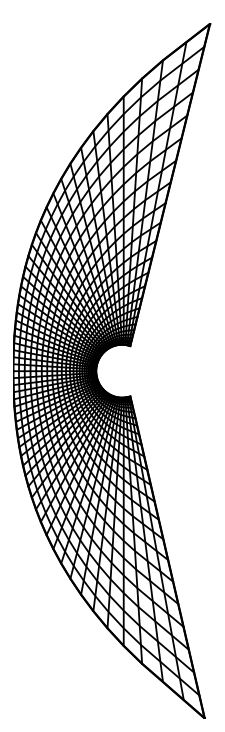

(a)

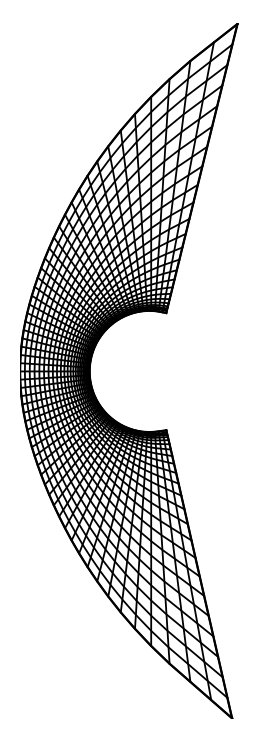

(b)

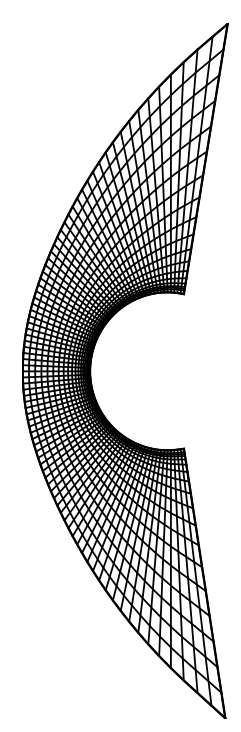

(c)

Fig. 9. Meshes in cylinder case. (a) $54 \times 20$, (b) $54 \times 20$, (c) $80 \times 14$

In terms of computational efficiency, the flux calculation of GRP spends only a little bit more time, say 5\%, than the first-order exact Riemann solver (Godunov). The computational cost of GRP flux is lower than GKS. At Mach number 2, the time ratio spending on the flux calculation only between GRP and GKS is about 5/7. However, if we include the reconstruction, the evaluation of CFL time step, the flow field updating and checking, and the residual evaluation, the costs of two codes are nearly the same. With the current computational power, the time spending on the flux evaluation in a code takes only a small fraction of total computational time in a software. Within the same order of computational cost, the accuracy of the modeling seems more important than using inaccurate cheaper flux 


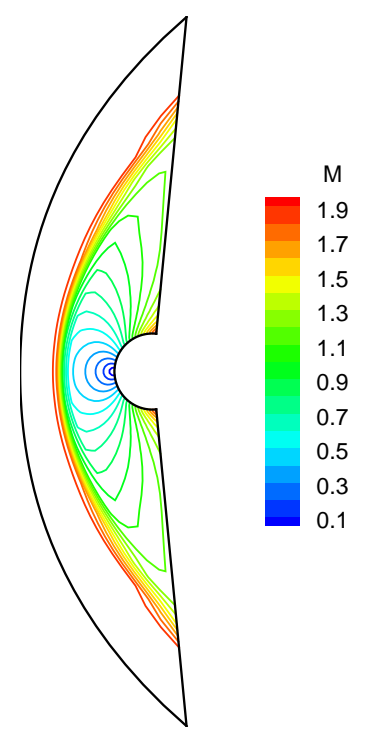

(a)

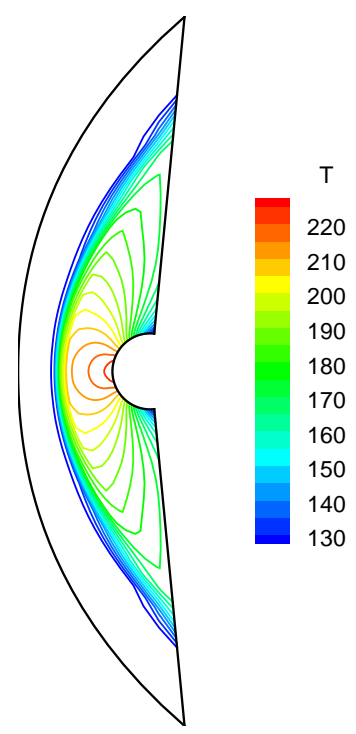

(c)

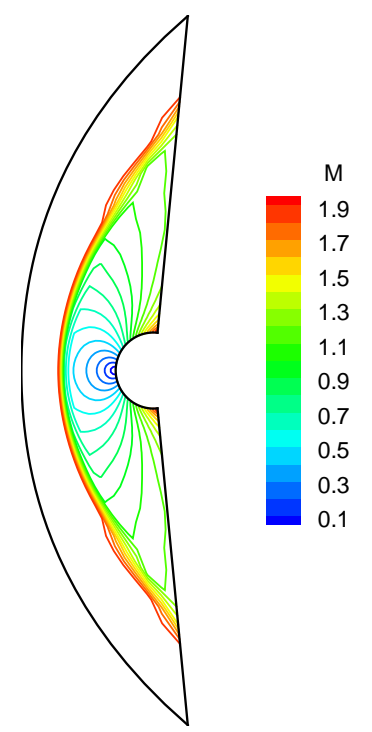

(b)

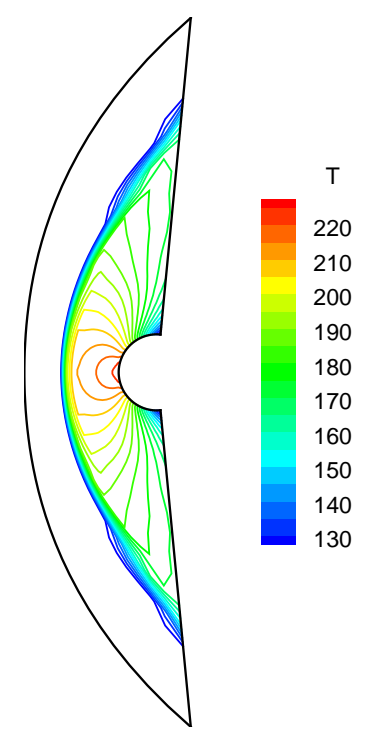

(d)

Fig. 10. $M=2$ case with mesh (a). The left column is computed by the GKS and the right by the GRP.

replacements. 


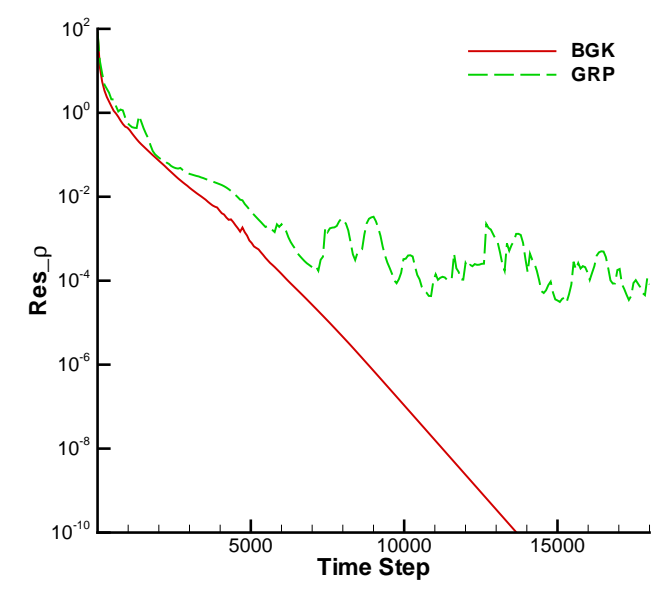

Fig. 11. $M=2$ case with mesh (a). Convergence residue distribution.

\section{Analysis}

In terms of Riemann solver, the GRP provides the most accurate flux function for the Euler equations under piecewise linearly discontinuous initial distribution. The wave interaction and modification of the characteristic waves due to spatial variation of initial flow variables have been fully included in GRP. The scheme used in this paper is probably the ultimate one people can construct under the piecewise discontinuous initial condition. Different from many other approximate Riemann solvers, the GRP is truthfully solving the Euler equations. This is also the reason why the current comparison is important. Through the numerical tests, we may answer the question about the validity of using and solving the Euler equations in a discretized space, even for the inviscid flow. The GKS solves the Boltzmann equation, but it presents accurate Euler solutions as well. In all tests, GRP and GKS are using the identical initial reconstruction, any difference in the simulation results is coming from the mechanism of constructing the flux function. Even with different underlying governing equations, the GRP and GKS have close similarity in the construction of local solution. Both schemes present a time-dependent flow evolution from an initially discontinuous data. In smooth regions, both methods go back to the traditional Lax-Wendroff method, where the spatial derivatives are used to get the temporal variation.

The GRP and GKS present different mechanism to describe the flow evolution from a discontinuity. For the GRP, in order to get all kinds of distinct waves in the generalized Riemann solution, it assumes that there is intensive particle collision and the gas sets down to the equilibrium state everywhere, even inside highly dissipative shock layer. This assumption is inconsistent with the real flow physics. The GKS first presents particle free transport process from an initially discontinuous data, then through the particle collision it generates the dissipative wave structure. With intensive particle collisions within a time step, a Navier-Stokes gas distribution function can be recovered from the GKS. The Euler solution is considered as a limiting case. The physical mechanism of the GKS can be hardly described using any macroscopic governing equation. The original BGK equation itself only provides a mecha- 


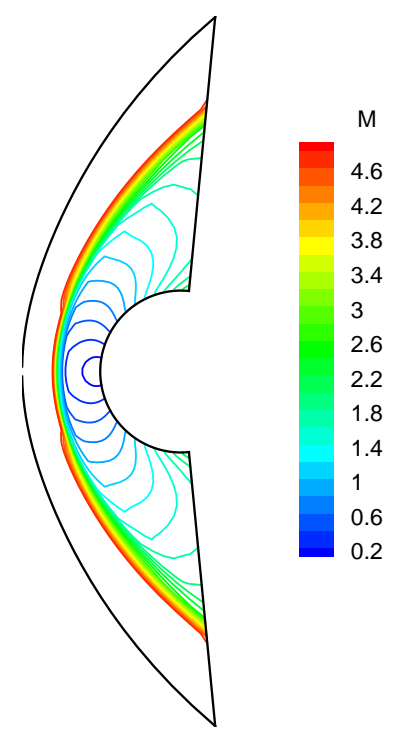

(a)

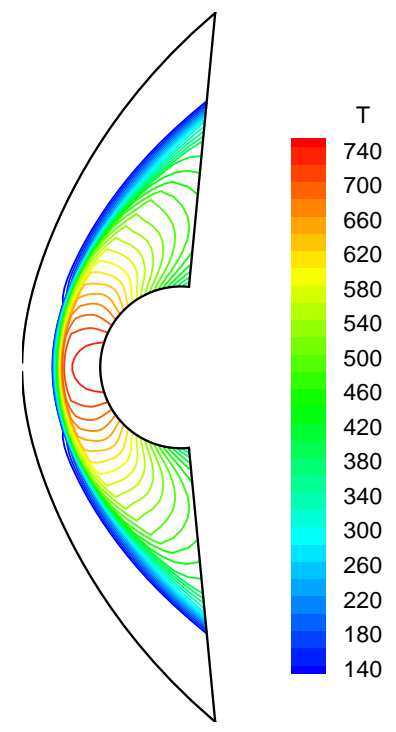

(c)

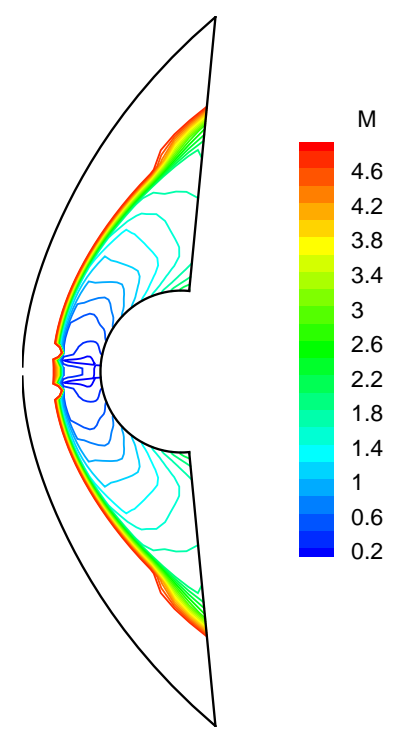

(b)

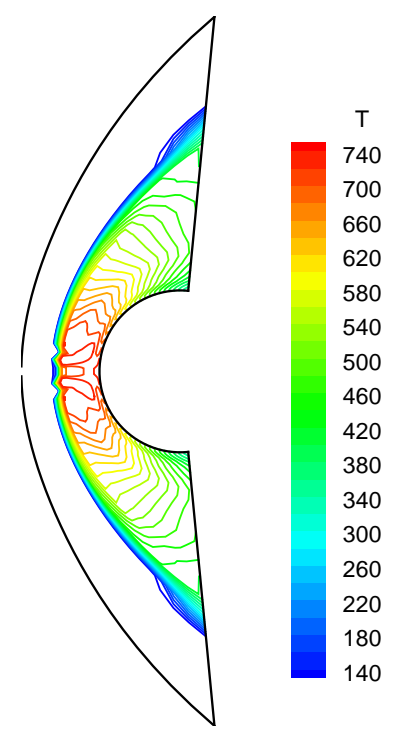

(d)

Fig. 12. $M=5$ case with mesh (b). The left column is computed by the GKS and the right by the GRP.

nism for the transport and collision on a scale of $\Delta x \rightarrow 0$ and $\Delta t \rightarrow 0$. Through the inclusion of initial discontinuity in a scale of $\Delta x \neq 0$ and $\Delta t \neq 0$, and the physical modeling in the scheme, the GKS provides a mechanism beyond BGK equation itself. 


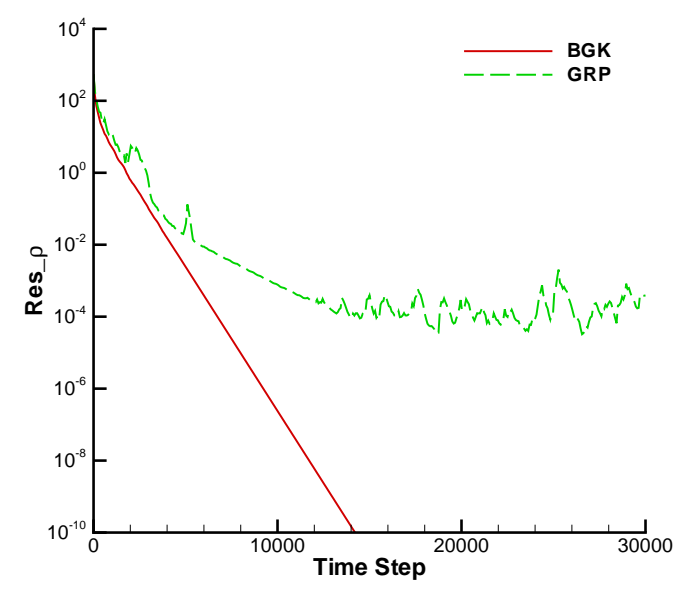

Fig. 13. $M=5$ case with mesh (b). Convergence residue distribution.

A numerical shock layer has to be considered as an enlarged physical shock structure. Since the shock thickness is on the order of particle mean free path, an enlarged numerical shock layer, i.e., across a few mesh size, needs a numerical cell size to be comparable with the pseudo-particle mean free path. Therefore, on the scale of mesh size, the non-equilibrium flow physics need to be taken into account in the gas evolution process in the discontinuous region. As we know, as a particle moves across a shock layer, there is only limited number of particles collisions. The non-equilibrium shock structure is constructed through the competition between particle free transport and collisions. This non-equilibrium process provides the appropriate dissipation for the smooth transition from one equilibrium upstream to another equilibrium downstream states. However, inside numerical shock layer the GRP replaces the non-equilibrium physical reality by an equilibrium one with the assumption of infinite number of particle collisions. An equilibrium state inside a shock layer, or the flux evaluation mechanism inside Riemann solution, cannot provide enough numerical dissipation, especially in multidimensional case. As a consequence, the use of the Euler equations itself in the flux modeling is not valid in the discontinuous region. The GKS follows the flow physics. The initial free transport, which provides the amount of dissipation, depends closely on the jump of initial discontinuity. This amount of dissipation can be hardly pre-assigned in a governing equation because the jump itself has numerical uncertainties, such as the use of different limiters. For a valid numerical scheme, theoretically we do not need to follow a precise physical shock structure in the scale of mesh size, but the gas evolution process has to be consistent with the physical one. The trigger of shock instability for GRP in high Mach number cases is due to the absence of non-equilibrium mechanism to provide "adjustable" dissipation in its flux function. In the smooth flow regions, due to intensive particle collision, the GKS will quickly evolve the solution into an equilibrium state, which recovers the inviscid Euler solutions.

Through this study, we may think of the possible direction for the further development of CFD algorithms. Since GRP is truthfully solving the Euler equations under the generalized initial condition, the absence of non-equilibrium mechanism in the Euler solutions shows that the Euler equations cannot be properly used as a governing equation for numerical fluid 


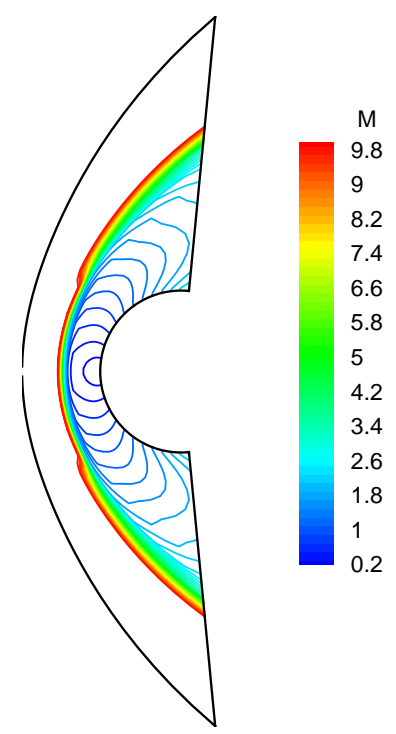

(a)

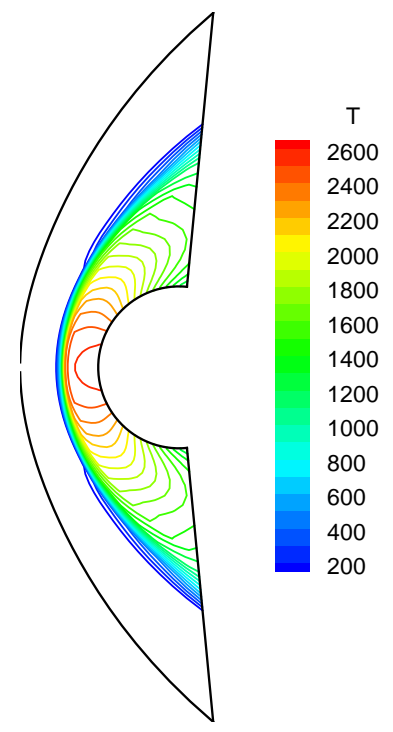

(c)

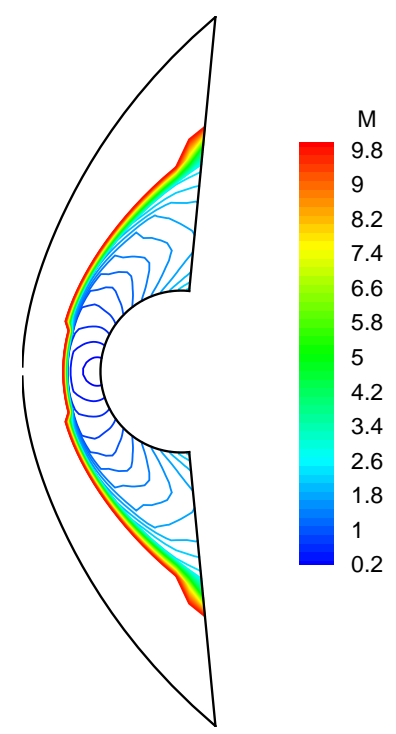

(b)

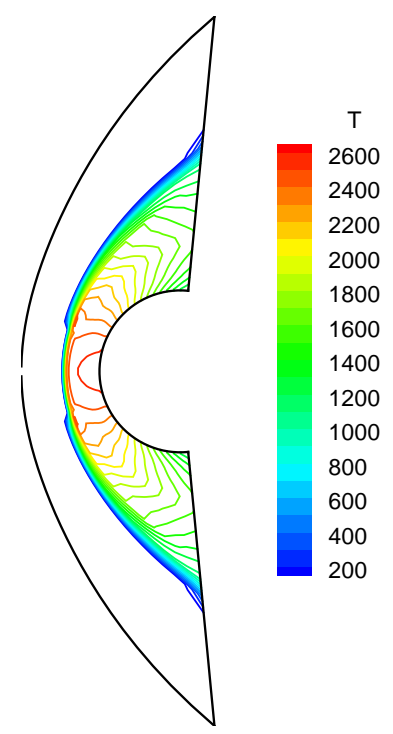

(d)

Fig. 14. $M=10$ case with mesh (b). The left column is computed by the GKS and the right by the GRP.

in a discretized space. Due to the limited cell resolution, any zero thickness discontinuity in the Euler solution will be enlarged numerically. As a result, the corresponding physical process has to be included in the numerical scheme. One may think of using the Navier- 


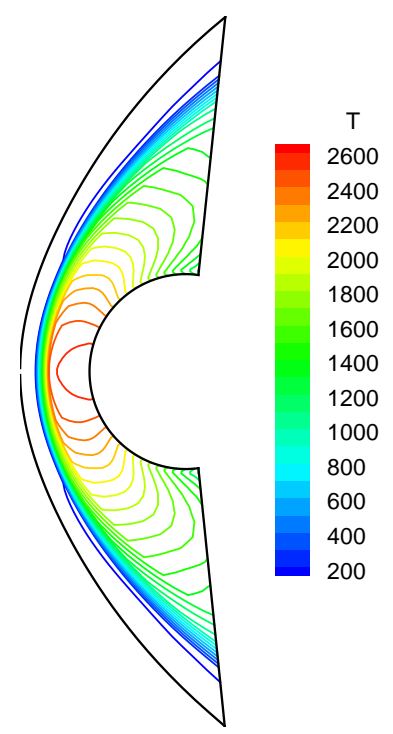

(a)

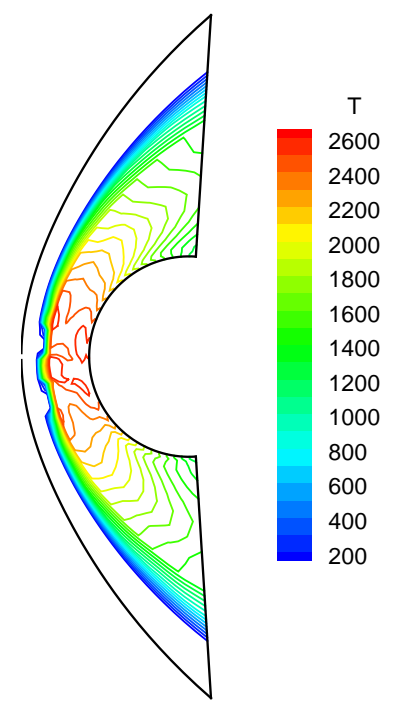

(b)

Fig. 15. $M=10$ case with mesh (c). The left is temperature distribution computed by the GKS and the right by the GRP.

Stokes equations with dissipative terms to capture the corresponding physical process inside the non-equilibrium layer. But, this cannot be valid as well, because the NS equations have only the physical dissipation, which have no any account on the scale of the dissipative region and the jump of the discontinuity. In other words, the dissipation provided in the governing equations should be able to adjust itself according to the locally reconstructed data.

The main idea we would like to deliver is that for a shock capturing scheme we need a correct physical mechanism to model the gas evolution from a discontinuity in the discretized space. The Euler equations do not provide such a correct physical mechanism. The real physics from a discontinuity is that the particles take free transport first, then particles collisions push the system towards to equilibrium state. Due to the discontinuity this process cannot be properly described by any well-defined macroscopic governing equation. On the other hand, even with a correct physical mechanism in a discretized space, it may be inappropriate to get such a governing equation because we can never allow the mesh size to approach to zero. The GKS constructs a physical process from a discontinuity with the help of the gas-kinetic BGK equation. Through the modeling in the scheme, the physics provided inside the GKS is much more rich than that the BGK equation itself provides. 


\section{Conclusion}

In this paper, we have made a detailed comparison between two well-developed numerical methods for compressible flow computations, i.e., the generalized Riemann solver and the gaskinetic scheme, by simulating many 1-D and 2-D test cases. The comparison is made in terms of the accuracy, efficiency, and robustness of each method. The present study indicates that the GRP and GKS can both be applied to inviscid compressible flow computations in 1D case, and can give comparable predictions. GRP is more computational efficient than GKS, but GKS is more robust than GRP. For the 2D blunt body simulations, the performance of GRP and GKS deviates. Like many other Riemann solvers, the GRP has intrinsic shock instability in the high Mach number flow computations. Since the initial reconstruction used in both GRP and GKS are identical, any difference is coming from the flux evaluation mechanism. Through the comparison of evolution mechanism in GRP and GKS, it is realized that, even for the inviscid flow, the Euler equations cannot provide a valid physical evolution model in the numerical shock layer. The direct modeling of the physical process in a discretized space is more important than the direct adaptation of well-defined macroscopic governing equations, which are only valid in a continuous space and time.

\section{Acknowledgements}

This paper is done when the first two authors visit Hong Kong University of Science and Technology, whose hospitality and support are greatly appreciated. J. Li is partially supported by the Key Program from Beijing Educational Commission (KZ200910028002), PHR(IHLB) and NSFC (10971142,11031001). Q.B. Li is partially supported by National Natural Science Foundation of China (Project No. 10872112). K. Xu is supported by Hong Kong Research Grant Council 621709 and RPC10SC11, National Natural Science Foundation of China (Project No. 10928205), National Key Basic Research Program (2009CB724101).

\section{References}

[1] M. Ben-Artzi, The generalized Riemann problem for reactive flows, J. Comput. Phys., 81(1989), 70-101.

[2] M. Ben-Artzi and J. Falcovitz, A second-order Godunov-type scheme for compressible fluid dynamics, J. Comput. Phys., 55 (1984), 1-32.

[3] M. Ben-Artzi and J. Falcovitz, An upwind second-order scheme for compressible duct flows, SIAM J. Sci. Stat. Comput., 7 (1986), 744-768.

[4] M. Ben-Artzi and J. Falcovitz, Generalized Riemann Problems in Computational Fluid Dynamics, Cambridge University Press, 2003. 
[5] M. Ben-Artzi and J. Li, Hyperbolic balance laws: Riemann invariants and the generalized Riemann problem, Numer. Math. 106 (2007), 369-425.

[6] M. Ben-Artzi, J. Li and G. Warnecke, A direct Eulerian GRP scheme for compressible fluid flows, J. Comput. Phys., 218 (2006), 19-34.

[7] P.L. Bhatnagar, E.P. Gross, and M. Krook, A Model for Collision Processes in Gases I: Small Amplitude Processes in Charged and Neutral One-Component Systems, Phys. Rev., 94 (1954), pp. 511-525.

[8] J.P. Boris And D.L. Book, Flux-corrected Transport. I. SHASTA, A Fluid Transport Algorithm that Works, J. Comput. Phys., 11 (1973), pp. 38-69.

[9] S. Chapman and T.G. Cowling, The Mathematical Theory of Non-Uniform Gases 3rd ed. (Cambridge University Press, Cambridge, 1970).

[10] B. Einfeldt, C.D. Munz, P. L. Roe, B. Sjögreen, On Godunov-type methods near low densities Journal of Computational Physics, Vol. 92 (1991), pp. 273-295.

[11] J. Falcovitz, G. Alfandary, and G. Hanoch. A 2-D conservation laws scheme for compressible ows with moving boundaries, J. of Comput. Phys., 138 (1997), 83-102.

[12] J. Falcovitz and A. Birman, A singularities tracking conservation laws scheme for compressible duct flows, J. Comput. Phys., 115 (1994), 431-439.

[13] S. K. Godunov, A finite difference method for the numerical computation and disontinuous solutions of the equations of fluid dynamics, Mat. Sb., 47 (1959), 271-295.

[14] E. Han, J.Q. Li and H.Z. Tang, Accuracy of the adaptive GRP scheme and the simulation of 2-D Riemann problems for compressible Euler equations, to apear in CICP, 2010.

[15] A. Kurganov and E. Tadmor, Solution of two-dimensional Riemann problems for gas dynamics without Riemann problem solvers, Numer. Meth. Part. Diff. Eqs., 18 (2002), 584-608.

[16] J. Li and G. Chen, The generalized Riemann problem method for the shallow water equations with bottom topography, Int. J. Numer. Methods Eng., 65 (2006), 834-862.

[17] J. Li, T. Zhang and S. Yang, The Two-dimensional Riemann Problem in Gas Dynamics, Addison Wesley Longman, 1998.

[18] Q.B. Li, S. Fu, and K. Xu, A compressible Navier-Stokes flow solver with scalar transport, J. Comput. Phys., 204 (2005), pp. 692-714.

[19] Q.B. Li, S. Fu, and K. Xu, Application of BGK scheme with kinetic boundary conditions in hypersonic flow, AIAA J., 43 (2005), pp. 2170-2176.

[20] X.D. Liu and P.D. Lax, Solution of two-dimensional Riemann problems of gas dynamics by positive schemes, SIAM J. Sci. Comput., 19 (1998), 319-340.

[21] T. Ohwada, On the construction of kinetic schemes, J. Comput. Phys. 177, pp. 156175 (2002).

[22] C.W. Schulz-Rinne, J.P. Collins, and H.M. Glaz, Numerical solution of the Riemann problem for two-dimensional gas dynamics, SIAM J. Sci. Comput., 14 (1993), 1394-1414. 
[23] H. Z. Tang and T. G. Liu, A note on the conservative schemes for the Euler equations, Journal of Computational Physics, Volume 218, Issue 2, (2006), Pages 451-459.

[24] E.F. Toro, Riemann Solvers and Numerical Methods for Fluid Dynamics: A Practical Introducition, Springer, 1997.

[25] B. van Leer, Towards the ultimate conservative difference scheme V, J. Comput. Phys., 32 (1979), 101-136.

[26] P. Woodward and P. Colella, The numerical simulation of two-dimensional fluid flow with strong shocks, J. Comput. Phys., 54 (1984), 115-173.

[27] K. Xu, A gas-kinetic BGK scheme for the Navier-Stokes equations and its connection with artificial dissipation and Godunov method, J. Comput. Phys. 171 (2001) 289-335.

[28] K. Xu, M.L. Mao, and L. Tang, A multidimensional gas-kinetic BGK scheme for hypersonic viscous flow, J. Comput. Phys., 203 (2005), pp. 405-421.

[29] T. Zhang and Y. Zheng, Conjecture on the structure of solutions of the Riemann problem for two-dimensional gas dynamics systems, SIAM J. Math. Anal., 21 (1990), 593-630. 